\title{
An early report of a screening program for colorectal cancer in Guangzhou, China
}

\author{
Yujing Fang ${ }^{1 \#}$, Binyi Xiao" ${ }^{1 \#, ~ J i a n h o n g ~ P e n g ~}{ }^{1}$, Huan Tian ${ }^{2}$, Fulong Wang ${ }^{1}$, Lingheng Kong ${ }^{1}$, Shiyong Lin ${ }^{1}$, \\ Jibin $\mathrm{Li}^{1}$, Qiying Su${ }^{2}$, Kunwu Luo ${ }^{2}$, Meixian $\mathrm{Ye}^{1}$, Qingjian Ou${ }^{1}$, Wenhua Fan ${ }^{1}$, Cong Li ${ }^{1}$, Junzhong Lin ${ }^{1}$, \\ Rongxin Zhang ${ }^{1}$, Wenhao Zhou ${ }^{1}$, Liren Li $^{1}$, Gong Chen ${ }^{1}$, Zhenhai Lu ${ }^{1}$, Xiaojun Wu ${ }^{1}$, Peirong Ding ${ }^{1}$, \\ Meichun Zheng ${ }^{1}$, Xia Yang ${ }^{1}$, Zhizhong Pan ${ }^{1}$, Desen Wan ${ }^{1}$
}

${ }^{1}$ Sun Yat-sen University Cancer Center, State Key Laboratory of Oncology in South China, Collaborative Innovation Center of Cancer Medicine, Guangzhou 510060, China; ${ }^{2}$ Dadong Street Community Health Service Center, Guangzhou 510000, China

Contributions: (I) Conception and design: D Wan, Z Pan, Y Fang; (II) Administrative support: D Wan, Z Pan; (III) Provision of study materials or patients: L Kong, S Lin, F Wang, H Tian, Q Su, K Luo, M Ye, Q Ou, W Fan, C Li, J Lin, R Zhang, W Zhou, L Li, G Chen, Z Lu, X Wu, P Ding, M Zheng, X Yang; (IV) Collection and assembly of data: B Xiao, J Peng; (V) Data analysis and interpretation: J Li, B Xiao; (VI) Manuscript writing: All authors; (VII) Final approval of manuscript: All authors.

\#These authors contributed equally to this work.

Correspondence to: Zhizhong Pan; Desen Wan. Department of Colorectal Surgery, Cancer Center, Sun Yat-sen University, 651 Dongfeng Road East, Guangzhou 510060, China. Email: Panzhzh@sysucc.org.cn; wands@sysucc.org.cn.

Background: We launched a screening program for colorectal cancer (CRC) in Yuexiu District, Guangzhou, China, in 2014. Here we aimed to report the early results of the program and evaluate the benefits of a screening questionnaire.

Methods: Residents aged between 50 and 74 were eligible for the screening. A questionnaire and two consecutive fecal immunological tests (FITs) were used as primary screening methods. Subjects who were positive for any of the two tests were referred for further examination with colonoscopy. Neoplasms were removed either colonoscopically or by colectomy. Atypical adenoma and CRC were defined as advanced neoplasms.

Results: A total of 6,971 residents in Dadong Street, Yuexiu District were screened with a questionnaire, and among them, 5,343 underwent at least one FIT. Four thousand and two hundred eleven (60.4\%) were female, and 2,760 (39.6\%) were male, with a median age of 62.0 years. Questionnaire and FITs identified 1,219 candidates for further examination with colonoscopy, among whom only 647 (53.1\%) comply. As of this writing, 623 colonoscopy results were obtained, among which 270 (43.3\%) had positive findings. The adenoma detection rate (ADR) was 43.3\% (270/623). The ADR was 43.3\% (270/623). Of the 270 patients, $10(3.07 \%)$ had CRC, 81 (30.0\%) had advanced adenoma, 178 had low-grade adenoma or other benign polyps, one had carcinoid. Except for three advanced CRC, all neoplasms detected were benign or in an early stage.

Conclusions: Our screening program help identified patients with colonic neoplasms at an early stage, precluding them from developing into the malignant disease. The addition of the questionnaire significantly increased the sensitivity of primary screening, while also decreasing the specificity. Long-term results should evaluate the social and economic benefits of this program.

Keywords: Polyps; colorectal cancer (CRC); early screening; fecal immunological test (FITs)

Submitted Aug 14, 2019. Accepted for publication Sep 03, 2019.

doi: 10.21037/atm.2019.09.75

View this article at: http://dx.doi.org/10.21037/atm.2019.09.75 


\section{Introduction}

Colorectal cancer (CRC) is a significant threat to public health. In 2018, there is an estimate of 1.8 million new incidences and 0.81 million deaths globally. CRC ranks third in terms of incidence and second in mortality, accounting for one in ten cancer cases and deaths (1). In China, CRC has become the fifth most common cancers in men and the third most common cancers in women, with an estimate of 37.6 new cases per year (2). While the incidence of CRC is on the rise in both China and most other countries in the world, it has been declining in the past two decades in the United States (3). Between 2004 and 2013, both the incidence and mortality of CRC in the USA declined at an annual rate of about 3\% (4). This trend is believed to result from the prevalence of CRC screening among high-risk adults (5).

Screening programs for the early detection of colorectal neoplasms have been launched in Haining (Zhejiang province), Tianjin, and Guangzhou (Guangdong province) to curb the upward trend of CRC incidence in China $(6,7)$. For economic reasons, these programs use fecal occult blood test (FOBT) and a specially designed questionnaire as primary screening methods before visual inspection with colonoscopy examination. Although their long-term effect on CRC control is yet to be seen, early reports have shown promising results. Jiang et al. found that $20 \%$ of the subjects in Haining city undergoing colonoscopy had one or more neoplastic lesions, most of which were benign polyps. The similar detection rate was observed in another program in Tianjin city.

In 2015, we launched a screening program for CRC in Guangzhou in consort with local Disease Control Center. Up to date, we have screened 6,971 eligible population with questionnaire and FOBT and performed 652 colonoscopy examinations. Here, we reported the early results of this program and evaluated the usefulness of the questionnaire.

\section{Methods}

\section{Screening protocol}

We initiated a population screening program for colorectal neoplasms together with Guangzhou CDC in Yuexiu District, Guangzhou, Guangdong Province, China, in 2014. The eligible population included residents aged between 50 and 74 years. As of Dec 2018, 6,971 patients have been screened. To reduce the cost and increase detection rate, we adopted a two-step screening method: subjects were first evaluated for the risk of colorectal neoplasms using questionnaires and FOBT simultaneously; those who were positive in any of the tests were then referred for further evaluation with colonoscopy.

\section{Primary screening}

The primary screening included a questionnaire and two fecal immunological tests (FITs). We used the same questionnaire as was used in other screening programs in China. It included sex, age, persistent symptoms of the gastrointestinal tract, history of cancer, familial cancer history, diet habit, drinking, and smoking. Subjects were defined as high-risk if they had a history of polyps, any first-degree relatives with CRC cancer, or met $\geq 2$ of the following conditions: chronic constipation or diarrhea, hematochezia, traumatic experience in the past 20 years, history of appendicitis, and history of cholecystitis.

FIT was used to detect occult blood in stools. Each subject was provided with two collection kits (supplied by $\mathrm{ABON}$, China), and required to collect 10 to $50 \mathrm{mg}$ stool twice in two consecutive weeks, following the manufacturer's recommendations. Samples were sent to local community health centers within 6 hours after collection. All subjects were required to undergo a second test regardless of the result of the first test.

\section{Colonoscopy examination}

Participants that were positive for primary screening were referred to Sun Yat-sen University Cancer Center or other medical centers for further examination with colonoscopy. Polyps of radius $<0.5 \mathrm{~cm}$ were resected colonoscopically during the examination, if possible. Any neoplasm $\geq 0.5 \mathrm{~cm}$ was biopsied first and proceeded with polypectomy or colectomy depending on pathological report and feasibility of endoscopic surgery. Atypical adenomas, carcinoma in situ, and CRC were referred to as advanced neoplasms.

\section{Statistics}

Associations between categorical variables were assessed by Chi-square test, Fisher's exact test, or Wilcoxon's rank-sum test, as appropriate. A two-sided $\mathrm{P}<0.05$ was considered statistically significant. All analyses were performed in R 
Table 1 Basic information

\begin{tabular}{|c|c|}
\hline Subsets & $\mathrm{N}(\%)$ \\
\hline \multicolumn{2}{|l|}{ Sex } \\
\hline Female & $4,211(60.4)$ \\
\hline Male & $2,760(39.6)$ \\
\hline \multicolumn{2}{|l|}{ Age, years } \\
\hline Median & 62.0 \\
\hline$\leq 60$ & $2,861(41.1)$ \\
\hline$>60$ & $4,104(58.9)$ \\
\hline \multicolumn{2}{|l|}{ Education } \\
\hline No education & $35(0.5)$ \\
\hline Primary school & $600(8.6)$ \\
\hline Middle school & $5,169(74.2)$ \\
\hline Undergraduate & $1,141(16.4)$ \\
\hline Graduate & $26(0.4)$ \\
\hline \multicolumn{2}{|c|}{ Questionnaire result } \\
\hline Positive & 894 (12.8) \\
\hline Negative & $6,077(87.2)$ \\
\hline \multicolumn{2}{|l|}{ FITs results } \\
\hline Positive & $377(8.3)$ \\
\hline Negative & $4,187(91.7)$ \\
\hline
\end{tabular}

Table 1 (continued)

(version 3.5.3).

\section{Results}

\section{Completion rates}

As shown in Table 1, a total of 6,971 residents from Dadong Street, Yuexiu District were screened by questionnaire, among whom 4,211 (60.4\%) were female, and 2,760 (39.6\%) were male. The median age was 62.0 years. $16.8 \%$ of the population screened had received higher education.

There were $894(12.8 \%)$ subjects positive for the questionnaire, among whom 740 underwent FIT as required, 25 asked for direct colonoscopy examination, the other 129 were lost. Three hundred seventy-seven (8.3\%) positive subjects were identified by FITs. The first and second FIT identified 253 and 232 of them, respectively. There were 76 subjects positive for both FITs. In total,
Table 1 (continued)

\begin{tabular}{|c|c|}
\hline Subsets & $\mathrm{N}(\%)$ \\
\hline \multicolumn{2}{|l|}{ FITs \& questionnaire } \\
\hline Positive & $1,219(17.5)$ \\
\hline Negative & $5,752(82.5)$ \\
\hline \multicolumn{2}{|l|}{ Colonoscopy testing } \\
\hline Yes & $647(53.1)$ \\
\hline No & $572(46.9)$ \\
\hline \multicolumn{2}{|l|}{ Colonoscopy results } \\
\hline Hyperplastic polyp & $16(6.3)$ \\
\hline Inflammatory polyp & $57(22.4)$ \\
\hline Adenoma & $170(66.7)$ \\
\hline Adenocarcinoma & $11(4.3)$ \\
\hline Carcinoid & $1(0.4)$ \\
\hline \multicolumn{2}{|l|}{ Pathology } \\
\hline Advanced cancer & $3(0.5)$ \\
\hline Early cancer & $5(0.8)$ \\
\hline CRC of unknown stage & $2(0.3)$ \\
\hline Advanced adenoma & $81(13.0)$ \\
\hline Other polyps & $178(28.6)$ \\
\hline Carcinoid & $1(0.2)$ \\
\hline Negative & $353(56.7)$ \\
\hline
\end{tabular}

FIT, fecal immunological test; CRC, colorectal cancer.

Questionnaire and FITs identified 1,219 candidates for further examination with colonoscopy.

\section{Colonoscopy examination}

Among the 1,219 candidates for a colonoscopy, only 647 (53.1\%) complied with the recommendation. Of them, $77.1 \%$ were positive for the questionnaire, and $36.8 \%$ were positive for FITs. Compliance rate was higher in patients $>60$ than in patients $\leq 60(61.1 \%$ vs. $38.9 \%)$, although it was not statistically significant $(\mathrm{P}=0.092)$. The rate was not associated with sex $(\mathrm{P}=0.619)$ and education $(\mathrm{P}=0.261)$ (Table 2).

The results of the colonoscopy are listed in Table 3. As of this writing, 623 colonoscopy reports were obtained, among which $270(43.3 \%)$ had positive findings. The adenoma detection rate (ADR) was $43.3 \%$ (270/623). Of the 
Table 2 Comparisons between patients with and without colonoscopy follow-up

\begin{tabular}{lccc}
\hline Name & Follow-up & No follow-up & P value \\
\hline Sex & & & 0.619 \\
Female & $388(60.0)$ & $351(61.4)$ & \\
Male & $259(40.0)$ & $221(38.6)$ & \\
Age & & & 0.092 \\
$\leq 60$ & $252(38.9)$ & $250(43.7)$ & \\
$>60$ & $395(61.1)$ & $322(56.3)$ & \\
Questionnaire results & & & 0.001 \\
Positive & $499(77.1)$ & $395(69.1)$ & \\
Negative & $148(22.9)$ & $177(30.9)$ & \\
FIT results & & & 0.093 \\
Positive & $161(36.8)$ & $216(42.1)$ & \\
Negative & $277(63.2)$ & $297(57.9)$ & \\
Higher education & & & \\
Yes & $156(24.1)$ & $154(26.9)$ & \\
No & $491(75.9)$ & $418(73.1)$ & \\
\hline
\end{tabular}

FIT, fecal immunological test.

270 patients, $10(3.07 \%)$ had CRC, 81 (30.0\%) had advanced adenoma, 178 had low-grade adenoma or other benign polyps, one had carcinoid. Except for three advanced CRC, all neoplasms detected were benign or in an early stage. Neoplasms were more likely to occur in male patients (56.9\% vs. $34.9 \%, \mathrm{P}<0.001$ ), patients of $>60$ ages $(49.6 \%$ vs. $35.1 \%, \mathrm{P}<0.001)$, and patients with chronic constipation (46.0\% vs. $34.9 \%, \mathrm{P}=0.017)$.

\section{Comparison of accuracies}

Table 4 shows the predictive performance of the questionnaire and FIT. When the detection rate was concerned, the sensitivities of the questionnaire, FITs and their combination were $0.578,0.475$, and 0.919 , respectively. The addition of the questionnaire to FITs improved sensitivity by more than $40 \%$, but it also decreased the specificity to 0.088 . We then looked into their positive predictive values (PPVs) for neoplasms, and found that PPVs of the questionnaire, FITs, and their combination were $0.397,0.532$, and 0.435 , respectively.

\section{Discussion}

Population screening for early detection of polyps is thought to be the main reason for the decline in CRC incidence in the US $(5,8)$. In this article, we presented preliminary results of a screening program for CRC in Yuexiu District, Guangzhou, China. We found that primary screening with questionnaire and FIT helped identify a considerable proportion of patients with benign polyps or CRC of early stage. Removing these neoplasms would hopefully help decrease CRC incidence in this area in the future.

Screening with colonoscopy is undoubtedly the most straightforward way to detect neoplasms on the colonic lumen. The problem is to whom we should recommend this test in order to make the best of resources. Take America as an example, to reach the goal of screening $80 \%$ of its eligible population with colonoscopy by 2024, 11 to 13 million colonoscopies would be needed annually in a colonoscopyonly program, but only 5.1 million would suffice when coupled with the FIT (9). In previous studies, FIT achieved a sensitivity of 0.8 and a specificity of 0.9 . Commitment to an annual test with FIT yielded comparable outcome to that with colonoscopy every 5 years $(10,11)$. In our study, two consecutive FITs yielded a detection rate of $8.3 \%$, which was similar to that reported in the US (7-10\%) and Tianjin $(5.8 \%)$ programs $(5,7)$. However, the sensitivity of FIT was only $47.5 \%$, which meant among 242 colonoscopically confirmed positive patients only 115 were detected by FITs. Several factors may be responsible for this. First, according to an US study, although the accuracy of FIT was superior to that of traditional Hemoccult II testing, it varied between brands $(12,13)$; also, methods of sample collection might have an impact on the result. Collins et al. found that specimens taken from spontaneously passed stools were better than those obtained by digital rectal examination (14). Participants in our study were not specifically guided in sample collection.

To further cut down the costs and improve sensitivity, we combined a high-risk screening questionnaire with the FIT as a primary screening regimen. In previous studies, this regimen showed better performance in detection rate and sensitivity than did FIT alone (6,15). In our study, the addition of questionnaire help increase sensitivity to as high as 0.919 , and PPVs for neoplasms to 0.435 , which were consistent with the results of Tianhe Guangzhou screening 
Table 3 Comparisons between participants with positive and negative colonoscopic findings

\begin{tabular}{|c|c|c|c|}
\hline Name & Positive & Negative & $P$ value \\
\hline Sex & & & $<0.001$ \\
\hline Female & $134(34.9)$ & $250(65.1)$ & \\
\hline Male & $136(56.9)$ & $103(43.1)$ & \\
\hline Age & & & $<0.001$ \\
\hline$\leq 60$ & $94(35.1)$ & $174(64.9)$ & \\
\hline$>60$ & $176(49.6)$ & $179(50.4)$ & \\
\hline Questionnaire results & & & 0.016 \\
\hline Positive & $156(39.7)$ & $237(60.3)$ & \\
\hline Negative & $114(49.6)$ & $116(50.4)$ & \\
\hline FIT questionnaire & & & 0.779 \\
\hline Positive & $248(43.5)$ & $322(56.5)$ & \\
\hline Negative & $22(41.5)$ & $31(58.5)$ & \\
\hline FIT results & & & $<0.001$ \\
\hline Negative & $127(37.9)$ & $208(62.1)$ & \\
\hline Positive & $115(53.2)$ & $101(46.8)$ & \\
\hline Chronic diarrhea & & & 0.213 \\
\hline Yes & $53(38.7)$ & $84(61.3)$ & \\
\hline No & $217(44.7)$ & 269 (55.3) & \\
\hline Chronic constipation & & & 0.017 \\
\hline Yes & $52(34.9)$ & $97(65.1)$ & \\
\hline No & $218(46.0)$ & $256(54.0)$ & \\
\hline Familial colorectal cancer & & & 0.954 \\
\hline Yes & $48(42.5)$ & $65(57.5)$ & \\
\hline No & $218(43.6)$ & $282(56.4)$ & \\
\hline Unknown & $4(40.0)$ & $6(60.0)$ & \\
\hline
\end{tabular}

FIT, fecal immunological test.

program. This questionnaire helped screen for patients with colonic neoplasms. However, it should be noted that the addition of the questionnaire also lead to a dramatic drop of specificity, meaning a considerable proportion of healthy subjects would be referred for unnecessary colonoscopy.

Moreover, when the questionnaire alone was evaluated, its sensitivity was 0.578 , and its specificity only 0.393 . Therefore, using a questionnaire to screening out ineligible subjects may seem economical, but it risks leaving out $40 \%$ of positive cases. Questionnaire alone was no substitute for the FIT, and its economic value should be reassessed.

In addition to screening cost, low adherence to screening protocol presents another challenge. As shown in Table 1, the compliance rate for one FIT was $76.6 \%$, and only $65.5 \%$ had completed two FITs. The rate of colonoscopy follow-up was even lower, with only $51.3 \%$ of the high-risk participants undergoing the procedure. Although the same challenge is also facing screening programs in the US, with the rate ranging from $50 \%$ to $80 \%$, the reasons for it may be different from ours. In the US, insurance bears a significant part of the responsibility, since it does not cover the cost of colonoscopy for those positive for FIT (5). In our study, we offered free colonoscopy tests for all eligible subjects; therefore, some other factors were responsible. We first assumed that education might be to blame, but compliance rates were not significantly different between subjects with Higher Education and those without $(\mathrm{P}=0.261)$. However, when we looked deeper into the data, we found that $77.1 \%$ of the participants undergoing colonoscopy were positive for the questionnaire, while the rate was only $36.8 \%$ for FITs, hinting that questionnaire, a self-evaluating tool had a stronger effect than laboratory-assessed FIT in persuading subjects of the necessity of colonoscopy. Thus, raising the public's awareness of the benefits and safety of colonoscopy may help improve compliance.

Several factors can be used as indicators of quality of colonoscopy, among which ADR, defined as the proportion of patients with adenoma or CRC among the population screened, is the most popular one. A target $\mathrm{ADR}$ is recommended to be $\geq 25 \%$. Otherwise, there may be missing polyps $(16,17)$. Our study yielded an ADR of $43.2 \%$ (270/625), significantly higher than recommended, suggesting an excellent quality of colonoscopy. The result was consistent with those of other screening programs conducted in the Chinese population, reflecting high operating skills of Chinese colonoscopists in these programs $(6,7,15,18-21)$.

\section{Conclusions}

Our screening program help identified patients with colonic neoplasms at an early stage, precluding them from developing into the malignant disease. Long-term results should evaluate the social and economic benefits of this program. 
Table 4 Sensitivities and specificities of questionnaire and FIT testing

\begin{tabular}{|c|c|c|c|c|c|c|}
\hline Colonoscopy & \multicolumn{2}{|c|}{ Questionnaire results } & \multicolumn{2}{|c|}{ FIT results } & \multicolumn{2}{|c|}{ FIT \& questionnaire } \\
\hline Positive & 156 & 114 & 115 & 127 & 248 & 22 \\
\hline Negative & 237 & 116 & 101 & 208 & 322 & 31 \\
\hline Sensitivities & \multicolumn{2}{|c|}{0.578} & \multicolumn{2}{|c|}{0.475} & \multicolumn{2}{|c|}{0.919} \\
\hline NPVs & \multicolumn{2}{|c|}{0.504} & \multicolumn{2}{|c|}{0.621} & \multicolumn{2}{|c|}{0.585} \\
\hline PPVs & \multicolumn{2}{|c|}{0.397} & \multicolumn{2}{|c|}{0.532} & \multicolumn{2}{|c|}{0.435} \\
\hline
\end{tabular}

FIT, fecal immunological test; NPV, negative predictive value; PPV, positive predictive value.

\section{Acknowledgments}

Funding: The study was funded by National Natural Science Foundation of China (No. 81871991, No. 81502459, and No. 81772595) and the Guangzhou Science and Technology Planning Projects (Health Medical Collaborative Innovation Program of Guangzhou) (grant No. 201803040019 and No. 201400000001-4).

\section{Footnote}

Conflicts of Interest: The authors have no conflicts of interest to declare.

Ethical Statement: The authors are accountable for all aspects of the work in ensuring that questions related to the accuracy or integrity of any part of the work are appropriately investigated and resolved. The institutional review board of Sun Yat-sen University Cancer Center approved this study and written informed consent were obtained from all patients. The authenticity of this article has been validated by uploading the key raw data onto the Research Data Deposit public platform (www. researchdata.org.cn), with the approval RDD number as RDDA2019001156.

\section{References}

1. Bray F, Ferlay J, Soerjomataram I, et al. Global cancer statistics 2018: GLOBOCAN estimates of incidence and mortality worldwide for 36 cancers in 185 countries. CA Cancer J Clin 2018;68:394-424.

2. Chen $W$, Zheng R, Baade PD, et al. Cancer statistics in China, 2015. CA Cancer J Clin 2016;66:115-32.
3. Sung JJ, Lau JY, Goh KL, et al. Increasing incidence of colorectal cancer in Asia: implications for screening. Lancet Oncol 2005;6:871-6.

4. Howlader N, Noone AM, Krapcho M, et al. SEER Cancer Statistics Review, 1975-2016. Available online: https://seer. cancer.gov/csr/1975_2016/

5. Smith RA, Andrews KS, Brooks D, et al. Cancer screening in the United States, 2017: A review of current American Cancer Society guidelines and current issues in cancer screening. CA Cancer J Clin 2017;67:100-21.

6. Cai SR, Zhang SZ, Zhu HH, et al. Performance of a colorectal cancer screening protocol in an economically and medically underserved population. Cancer Prev Res (Phila) 2011;4:1572-9.

7. Zhao L, Zhang W, Dongwang MA, et al. Analysis of colorectal cancer screening practices in the general population of Tianjin. Chin J Clin Oncol 2015;42:760-4.

8. Hardcastle JD, Chamberlain JO, Robinson MH, et al. Randomised controlled trial of faecal-occult-blood screening for colorectal cancer. Lancet 1996;348:1472-7.

9. Joseph DA, Meester RG, Zauber AG, et al. Colorectal cancer screening: Estimated future colonoscopy need and current volume and capacity. Cancer 2016;122:2479-86.

10. Lee JK, Liles EG, Bent S, et al. Accuracy of fecal immunochemical tests for colorectal cancer: Systematic review and meta-analysis. Ann Intern Med 2014;160:171.

11. Bibbins-Domingo K, Grossman DC, Curry SJ, et al. Screening for Colorectal Cancer: US Preventive Services Task Force Recommendation Statement. JAMA 2016;315:2564-75.

12. Imperiale TF, Ransohoff DF, Itzkowitz SH, et al. Fecal DNA versus fecal occult blood for colorectal-cancer screening in an average-risk population. $\mathrm{N}$ Engl J Med 2004;351:2704-14. 
13. Daly JM, Bay CP, Levy BT. Evaluation of fecal immunochemical tests for colorectal cancer screening. J Prim Care Community Health 2013;4:245-50.

14. Collins JF, Lieberman DA, Durbin TE, et al. Accuracy of screening for fecal occult blood on a single stool sample obtained by digital rectal examination: A comparison with recommended sampling practice. Ann Intern Med 2005;142:81-5.

15. Li W, Zhao LZ, Ma DW, et al. Predicting the risk for colorectal cancer with personal characteristics and fecal immunochemical test. Medicine (Baltimore) 2018;97:e0529.

16. Pohl H, Robertson DJ. Colorectal cancers detected after colonoscopy frequently result from missed lesions. Clin Gastroenterol Hepatol 2010;8:858-64.

17. Rex DK, Schoenfeld PS, Cohen J, et al. Quality Indicators for Colonoscopy. Am J Gastroenterol 2015;110:72-90.

Cite this article as: Fang $\mathrm{Y}$, Xiao B, Peng J, Tian H, Wang F, Kong L, Lin S, Li J, Su Q, Luo K, Ye M, Ou Q, Fan W, Li C, Lin J, Zhang R, Zhou W, Li L, Chen G, Lu Z, Wu X, Ding P, Zheng M, Yang X, Pan Z, Wan D. An early report of a screening program for colorectal cancer in Guangzhou, China. Ann Transl Med 2019;7(21):604. doi: 10.21037/atm.2019.09.75
18. Jiang CX, Wang XP, Shen YZ. Comparative study of two rounds of colorectal cancer screening in 80, 000 targeted population in Haining. Zhonghua Zhong Liu Za Zhi 2017;39:707-11.

19. Yongzhou S, Huang Y, Lijuan Z, et al. A retrospective cohort study of 320 thousand subjects of colorectal cancer screening in Haining City. Zhonghua Zhong Liu Za Zhi 2015;37:317-20.

20. Li Y, Liu HZ, Liang YR, et al. Analysis of community colorectal cancer screening in 50-74 years old people in Guangzhou, 2015-2016. Zhonghua Liu Xing Bing Xue Za Zhi 2018;39:81-5.

21. Liao Y, Li S, Chen C, et al. Screening for colorectal cancer in Tianhe, Guangzhou: Results of combining fecal immunochemical tests and risk factors for selecting patients requiring colonoscopy. Gastroenterol Rep (Oxf) 2018;6:132-6 